\title{
Differences in feeding habits influence the growth performance and feeding efficiencies of African catfish (Clarias gariepinus) and lemon fin barb hybrid (Hypsibarbus wetmorei $\hat{\sigma} \times$ Barboides gonionotus + ) in a glycerol-based biofloc technology system versus a recirculating system
}

\begin{abstract}
African catfish (Clarias gariepinus) and lemon fin barb hybrid (LFBH) (Hypsibarbus wetmorei $\widehat{\sigma} \times$ Barbodes gonionotus + ) were cultured in either a biofloc technology (BFT) system or an individual recirculating aquaculture system (RAS) and their survival, growth, feeding efficiencies and biochemical composition were then compared after 8 weeks. LFBH and African catfish were chosen based on their different feeding habits. In the BFT treatments, glycerol was added to create a carbon to nitrogen ratio of 15 , while the RAS system consisted of a fine mesh mechanical and biological filter. Each of the four treatments was triplicated, with each replicate consisting of 20 African catfish $(0.98 \pm 0.05 \mathrm{~g})$ or LFBH $(1.77 \pm 0.02 \mathrm{~g})$. Water quality and biofloc formation were examined weekly while the biofloc proximate composition was measured in weeks 5 and 8. During weeks 6,7 and 8 the total viable bacterial colony forming units (CFU) were quantified. Regardless of species, biofloc formation was similar but the crude protein, lipid and ash significantly decreased from week 5 to week 8. However, biofloc crude protein and ash were significantly higher in the LFBH treatment. Total viable CFU were significantly higher $(P<0.05)$ in BFT compared to RAS, with LFBH having significantly more $(P<0.05)$ than African catfish. The proximate composition of the fish were unaffected by either system. Implementing BFT significantly $(P<0.05)$ improved both African catfish and LFBH growth, but this improvement was substantially higher for LFBH (41.6\%) than for African catfish (7.6\%). This may be due to a better ability of LFBH to consume smaller particles. Preliminary results also indicate that the nutritional composition of bioflocs could be influenced by biofloc age, which could have implications to solids management.
\end{abstract}

Keyword: Lemon fin barb hybrid; African catfish; Biofloc; Biofloc quality; Feeding habits; Recirculating aquaculture system 